\title{
Online-Konsultation wurde berufsrechtlich der Weg geebnet
}

\section{Videosprechstunde darf persönlichen Kontakt nicht ersetzen}

\author{
Die Videosprechstunde ist auf dem Weg in die Regelversorgung. Jetzt \\ wurden technische Anforderungen veröffentlicht, ab Juli 2017 soll es \\ eine eigene EBM-Ziffer geben. Alles andere als unmissverständlich sind \\ jedoch die berufsrechtlichen Rahmenbedingungen.
}

$\mathrm{V}$ iel Technik, wenig Konkretes zum eigentlichen Ablauf: Mit der „Vereinbarung über die Anforderungen an die technischen Verfahren zur Videosprechstunde gemäß $\$ 291 \mathrm{~g}$ Absatz 4 SGB V“, so der komplette Titel des jüngsten Vertragswerks der Selbstverwaltung, haben Kassenseite und Ärzteschaft den ersten Schritt zur Etablierung des digitalen Arztbesuchs nach dem E-Health-Gesetz gemacht.

Vertragsgegenstand, heißt es, sei die „synchrone Kommunikation zwischen einem Arzt und einem ihm bekannten Patienten (...) im Sinne einer OnlineVideosprechstunde in Echtzeit". Damit wird gleich eingangs des Paragrafenwerks auf die Berufsrechtsvorgabe Bezug genommen, wonach individuelle Behandlungen und Beratungen „nicht ausschließlich über Print- und Kommunikationsmedien" erfolgen dürfen.

Zur Erinnerung: Diesen Passus der Musterberufsordnung ( $\$ 7$ Abs. 4) präzisierte die Bundesärztekammer in einer Erläuterung zuletzt dahingehend, dass bei Fernbehandlungen ein persönlicher Vorkontakt stattgefunden haben muss. Diagnosen und Therapieempfehlungen „unbekannter Patienten“ seien berufsrechtswidrig. Präzisiert wird „unbekannt" nach vier Kriterien. Der behandelnde Arzt hat:

_keine Kenntnis über wichtige Vorbefunde des Patienten,

- keine medizinischen Informationen zum Krankheitsverlauf,

_keine Kenntnisse über das soziale Umfeld des Patienten,
— selbst den Patienten noch nicht körperlich untersucht.

Die KBV weist zudem darauf hin, dass die Online-Konsultation den persönlichen Arzt-Patient-Kontakt „nicht ersetzen darf" und nur „bei einer bereits begonnenen Behandlung ergänzend eingesetzt werden kann". Als Beispiele werden Gespräche zur Kontrolle des Behandlungsverlaufs genannt oder um eine Medikationsänderung zu erläutern. Ein solches Präjudiz enthält das E-HealthGesetz allerdings nicht.

Erst kürzlich hatte die Ärztekammer Baden-Württemberg berufsrechtlich den Weg für Modellprojekte frei gemacht, in denen ,ärztliche Behandlungen ausschließlich über Kommunikationsnetze durchgeführt werden“.

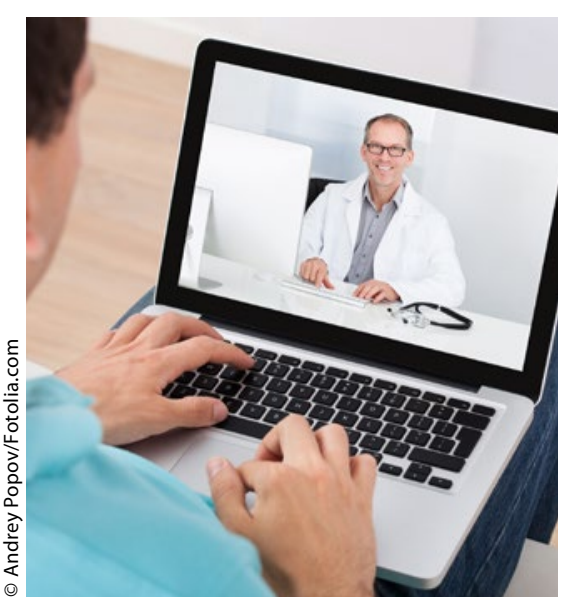

Laut KBV darf die Online-Konsultation den persönlichen Arzt-Patient-Kontakt nicht ersetzen sondern nur ergänzen.
Der Lübecker Dienstleister Patientus, der Video-Sprechstunden in Kooperation mit Kassen, Kliniken, Arztnetzen und Berufsverbänden organisiert, rechnet damit, dass ,in Zukunft weitere Landesärztekammern Änderungen am Fernbehandlungsverbot vornehmen werden", wie ein Unternehmenssprecher auf Anfrage erklärte.

In den jetzt vereinbarten Anforderungen an Videosprechstunden wird vorgegeben, dass Videosprechstunden in geschlossenen Räumen mit , angemessener Privatsphäre" stattzufinden haben. Aufzeichnungen sind während des Onlinekontakts nicht erlaubt.

Vertragsärzte müssen vom Patienten eine schriftliche Einwilligung in die Erhebung, Verarbeitung und Nutzung seiner Gesundheitsdaten einfordern, „die der Patient jederzeit widerrufen kann“. Und: Delegationsfähig ist die Videosprechstunde nicht, sie darf nur vom Vertragsarzt durchgeführt werden.

\section{Lange Liste mit Forderungen an Videodienstanbieter}

Damit hat es sich dann aber auch schon mit den Anforderungen, die Vertragsärzte zu beachten haben. Weitaus länger ist die Liste mit Forderungen an Videodienstanbieter. Die müssen unter anderem dafür sorgen, dass sich Patienten ohne Account zu einer Sprechstunde anmelden können und dass der Klarname des Patienten für den Arzt am anderen Ende der Leitung zu erkennen ist. Die Übertragung der Videosprechstunde darf nicht über einen zentralen Server führen, sondern muss direkt von einem zum anderen Rechner erfolgen (,peer-topeer"). Sämtliche Inhalte müssen verschlüsselt übertragen werden und nicht zuletzt dürfen Videodienstanbieter keinerlei Werbung zur Sprechstunde schalten.

Christoph Winnat 